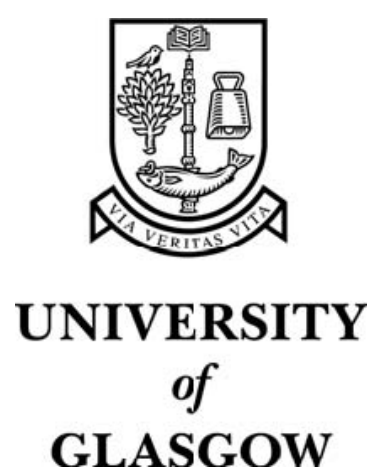

Gharai, L. and Lehman, T. and Saurin, A. and Perkins, C. (2006) Experiences with high definition interactive video conferencing. In, IEEE International Conference on Multimedia and Expo, 9-12 July 2006, pages pp. 433-436, Toronto, Canada.

http://eprints.gla.ac.uk/3585/ 


\section{EXPERIENCES WITH HIGH DEFINITION INTERACTIVE VIDEO CONFERENCING}

\author{
Ladan Gharai Tom Lehman \\ Information Sciences Institute \\ University of Southern California
}

\author{
Alvaro Saurin Colin Perkins \\ Department of Computing Science \\ University of Glasgow
}

\begin{abstract}
We review the design and implementation of UltraGrid, a new high definition video conferencing system, and present some experimental results. UltraGrid was the first system to support gigabit rate high definition interactive video conferencing on commodity systems and networks, and we present measurements to illustrate behavior of production networks subject to such real time traffic. We illustrate the benefits of hybrid IP/provisioned optical networks over best effort IP networks for this class of traffic, and motivate the development of congestion control algorithms for interactive conferencing on best effort IP networks.
\end{abstract}

\section{INTRODUCTION}

We review the design and architecture of UltraGrid [17], a new high definition video conferencing system. UltraGrid is the first in a new breed of systems capable of supporting high definition video over IP, that has greatly evolved the state of the art in video conferencing systems compared to both early research prototypes (e.g. [12]) and also modern commercial offerings.

We present measurement studies to show how modern, appropriately provisioned, IP networks and hybrid IP/optical networks can support even the most demanding of real-time applications. Our data shows how the timing and scheduling properties of modern networks and operating systems affect application performance, and how existing real time transport protocols allow applications to compensate. We demonstrate that both best effort IP and hybrid IP/optical networks provide a solid basis for high performance real-time applications, and validate the design of the Real-time Transport Protocol [19, 2] and modern network architectures.

The outline of this paper is as follows: we present an overview of the aims of the UltraGrid project in section 2, and review the system design in section 3 . In section 4 we present a performance evaluation of UltraGrid on both pure IP and hybrid IP/Optical network paths, comparing the two architectures to understand their relative performance and to motivate future work on congestion control and on network design. Related work is described in section 5, and future work and conclusions are discussed in section 6.

\section{OVERVIEW OF THE ULTRAGRID SYSTEM}

Our goal in developing UltraGrid was to demonstrate that modern end-systems and well engineered IP networks can support ultra high quality conferencing environments. To this end, UltraGrid provides low latency, high definition video; high quality audio and large screen displays enhance the sense of presence, creating a realistic conferencing environment.

UltraGrid supports both standard and high definition (HD) interactive video conferencing, using readily available hardware. Both progressive ("720p") and interlaced ("1080i") HD video is supported. Video may be transmitted using an uncompressed format if network capacity is available (either at $1.2 \mathrm{Gbps}$ for standard format HD video, or at $980 \mathrm{Mbps}$ with an alternative HD format). In addition, a range of video codecs are supported to allow adaptation to lower rates at the expense of some increase in latency and reduction in quality. UltraGrid is typically used in conjunction with AccessGrid [10], or some other session initiation framework, to provide the complete conferencing experience shown in Figure 1.

In addition to interactive conferencing, UltraGrid can be used for general purpose HD distribution and visualization. The sender converts stored file content or live SMTPE 292M [13] high-definition video, as produced professional cameras and production equipment, into an RTP packet stream for distribution across a variety of IP-based networks, and allows the receiver to exactly reconstruct the original signal. Unlike content distribution networks, our design seeks to minimize latency, and allows UltraGrid to be used for interactive video conferencing, data visualization and on-line video editing.

\section{DESIGN AND IMPLEMENTATION}

A major influence on the design of UltraGrid was to build a system that can be replicated by others, with an objective of significantly evolving the quality of baseline interactive video conferencing systems. To this end, we built UltraGrid from commercial off the shelf components, make use of standard protocols and codecs (additional RTP Profiles and Payload Formats were developed and published through the standards process as needed $[4,5,6])$, and made our software available under an open source license. In the following we describe the design of our software, and outline hardware requirements. 


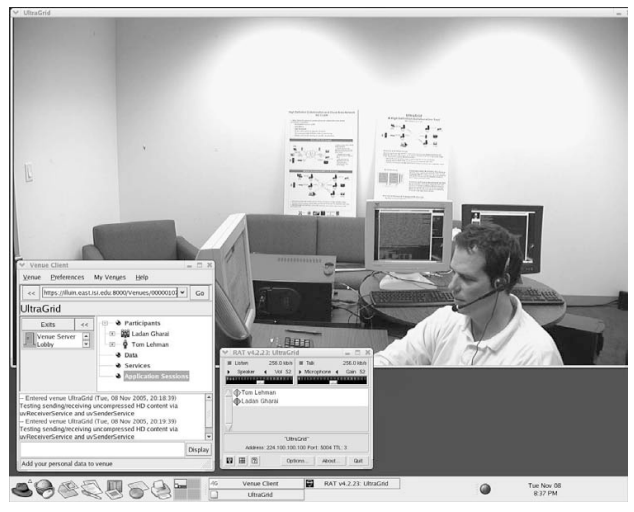

Fig. 1. UltraGrid in action.

\subsection{Software Architecture}

We present the high-level design of the UltraGrid software in Figure 2. The system is modular, allowing experiments with different capture and display devices, codecs and transport protocols. It currently supports capture and display of 8- and 10-bit high definition video and DV format standard definition video, codecs for uncompressed, DV and motion JPEG formats, and a complete implementation of the RTP transport protocol supporting IPv4, IPv6 and multicast.

Transmission and reception are implemented as four separate threads to improve the system performance and responsiveness. The sender uses one thread for grabbing and encoding, and a second thread for transmission and control. RTP packetization is done according the RFC 4175 [6]. In the same way, the receiver de-couples the reception and rendering of frames using two threads. The playout buffer has been designed according to the principles set forth in [16], in order to reduce effects of jitter and packet loss. Limited congestion control is present [4], but was not used since we seek to compare network performance, not congestion response.

\subsection{Hardware Requirements}

UltraGrid nodes are built from commercially available components. Nodes comprised Dual Xeon EM64T processors on Super Micro PCI-X mother boards, and ran a mixture of Fedora Core 3 or 4 (Linux 2.6.12 kernel) operating systems.

HD capture and/or playout uses HDstation or Centaurus cards from Digital Video Systems (DVS) [20]. These are used to capture the HD video, and to regenerate SMPTE-292M output at the receiver (it is also possible to display HD video directly in a window on the workstation monitor, Figure 1). For DV capture, an IEEE 1394 interface is required.

We use either gigabit Ethernet (integrated on the motherboard) or 10 gigabit Ethernet cards (Chelsio N110 [3]). An alternative approach is to use bonded gigabit Ethernet cards to support data rates up to 2 Gbps (such support is under development for UltraGrid [8]).

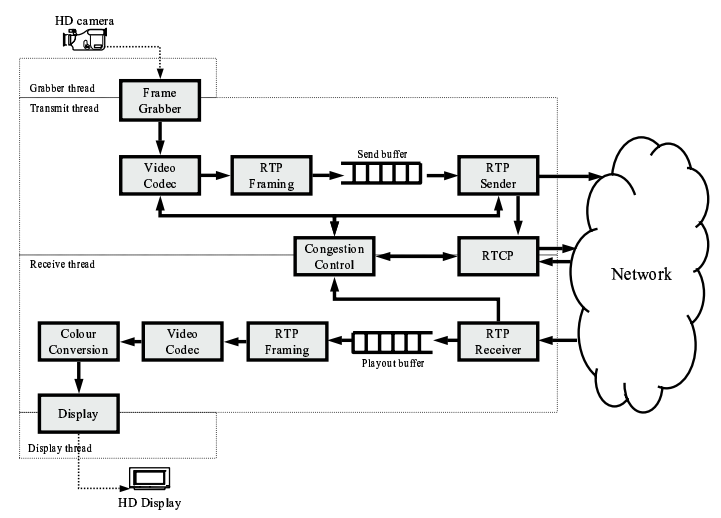

Fig. 2. UltraGrid software architecture.

\section{SYSTEM PERFORMANCE EVALUATION}

We conducted numerous local- and wide-area experiments to demonstrate correct operation of UltraGrid and to investigate the performance of IP and hybrid IP/optical networks.

\subsection{Local Area Tests}

Initial experiments used two nodes connected back to back via a direct optical 10 gigabit Ethernet link. Uncompressed 720p HD video flows with 8800 bytes packet sizes were exchanged between these nodes for 10 minutes: over 10 million packets were transmitted at a data rate of $1.2 \mathrm{Gbps}$ with no packet loss or reordering. This demonstrates that UltraGrid can support full rate HD video when not limited by the network, proving the end systems are not a performance bottleneck.

This experiment was repeated using the metropolitan area network from the DRAGON project [11]. As shown in Figure 3, this network has both an optical path and a number of Ethernet segments. Once again, tests were run for 10 minute periods using uncompressed HD video at 1.2 Gbps. Performance was essentially identical to the back to back tests, as is expected from a managed over provisioned optical network.

\subsection{Wide Area Tests}

Wide area tests were conducted during the Super Computing 2005 conference. An interactive HD video conference was run between the conference exhibit floor in Seattle, WA and ISI East in Arlington, VA for the duration of the conference (see Figure 4), running media flows over both the Internet2 Abilene best effort IP network, and the guaranteed capacity Hybrid Optical Packet Infrastructure (HOPI) circuit switched path. Individuals at ISI East were able to talk with participants at the conference via $720 \mathrm{p}$ format HD video. High quality low latency video, large displays and strategic positioning of cameras provided an effective sense of presence.

Connections to the best effort IP network were via gigabit Ethernet, and this limited us to using colour-subsampled 


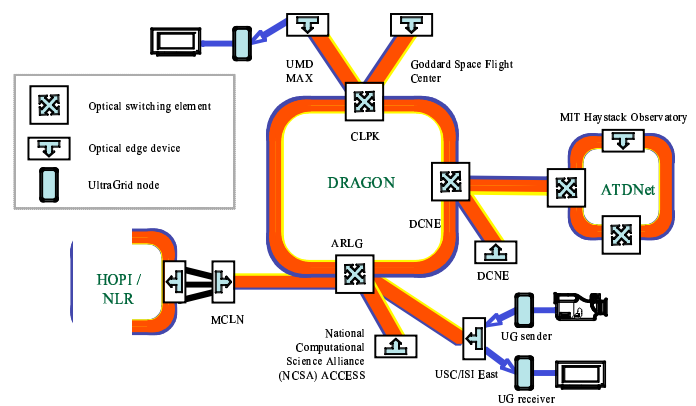

Fig. 3. Local testing of UltraGrid on the DRAGON network.

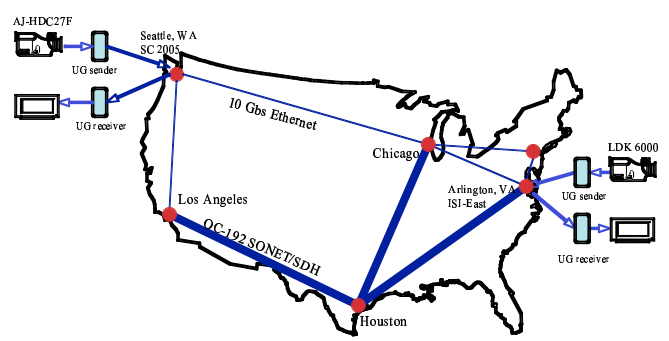

Fig. 4. UltraGrid 2005 Super Computing demonstration.

HD video at 980 Mbps. We used 8800 octet packets, and the network round trip time on this path was $77 \mathrm{~ms}$. Connection to HOPI was via a 10 gigabit Ethernet path, allowing us to use full 1.2 Gbps uncompressed HD video. We used 8800 octet packets, and the network round trip time on this path as $66 \mathrm{~ms}$. On both paths we measured performance for several 10 minutes periods over the duration of the week, and also captured packet traces to allow us to study packet loss and timing, along with various application level metrics. Due to lack of space here, we report only on loss and timing results.

We did not observe any packet loss on the HOPI path, as expected from a dedicated provisioned optical backbone path. The best effort IP path over was generally loss free, but showed occasional packet loss up to $0.04 \%$ due to transient congestion on the path (which is shared with the production IP traffic of Internet2, over which we have no control).

We plot the distribution of inter-packet interval (IPI) at source on the best effort IP path in Figure 5(a) and on the provisioned HOPI path in Figure 5(b). As noted previously, the best effort path is connected via a gigabit Ethernet link. This link has a higher IPI than the HOPI path. The IPI distribution at the receiver is shown in Figure 6. Packets traversing HOPI paths maintain similar distribution and inter-arrival times as at the sender, while packets sent over the best-effort IP path experience a long tail distribution and more dispersion (our RTP playout buffer can trivially compensate for this dispersion). This is to be expected from a provisioned path versus a best effort data path, and is confirmed by our data.

An interesting phenomena we note on the best effort path is a reduction in mean IPI at the receiver (Figure 6(a)) when compared to the mean IPI at the sender (Figure 5(a)). We speculate that this is due to a network architecture where the core network and receiver are faster than the sender access network, causing packets in a flow to be bunched at intermediate routers. The IPI plots in Figure 6(a) show the effects of most packets being clocked out at $10 \mathrm{Gbps}$ line rate, matching the pure 10Gbps environment in Figures 5(b) and 6(b), but a long tail due to competing traffic. Further study of the reasons for this behavior is ongoing.

While both best effort and provisioned paths offer good performance, it appears that a hybrid IP/optical network such as HOPI can offer more predictable loss and reduced jitter. The reduction in network timing jitter is not significant for applications such as ours, but the ability to control packet loss caused by background traffic or other video conferences is important since it may reduce the need for, or change the type of, congestion control which must be implemented.

\section{RELATED WORK}

Experiments with HD video over IP have been underway for several years. Initial work relied on special purpose hardware, such as the Universal Network Access System developed by Tektronix in conjunction with us and with the University of Washington [21,5] and the system developed by NTT [9, 7]. Both demonstrated full rate, $1.485 \mathrm{Gbps}$, transport of HDTV over IP networks in 2001.

Advances in host and network technology (predicted in our earlier work [18]) now allow full rate HD systems to be built from commodity hardware. UltraGrid was the first such system, and has been widely used as a base for research by other groups $[15,8,1]$. More recent work from the University of Washington [14] appears to offer some similar features.

\section{CONCLUSIONS AND FUTURE DIRECTIONS}

Our experiments demonstrate that both native, best effort, IP networks and hybrid IP/optical networks can be engineered to support even the most demanding real-time applications, with performance far exceeding that of commercial IP-based video distribution systems. We show that provisioned optical paths can be used as a substrate for IP networks, reducing packet loss, queuing delays and timing jitter compared to best effort paths. We also observe that applications can effectively characterize and compensate for the timing properties of best effort IP networks, but that congestion control for real-time applications on shared paths is an open research problem.

The contributions of our work are two-fold: 1) we have demonstrated the relative performance of hybrid IP/optical network infrastructure compared to the native IP service when used for real time applications; and 2) we have demonstrated that extremely high performance real time applications can be successfully deployed on modern networks. Our ongoing 
(a) Sender IPI: 1 Gbps NIC (best effort IP path)

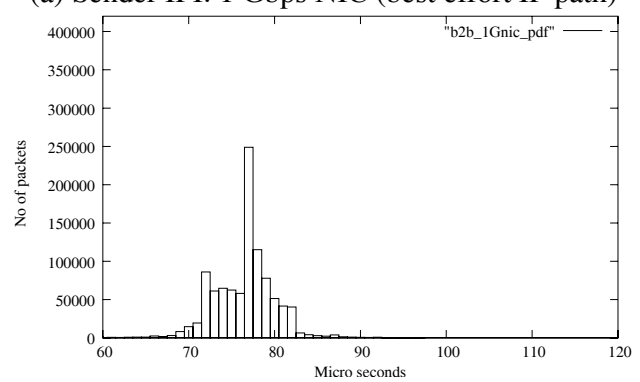

(b) Sender IPI: 10 Gbps NIC (provisioned HOPI path)

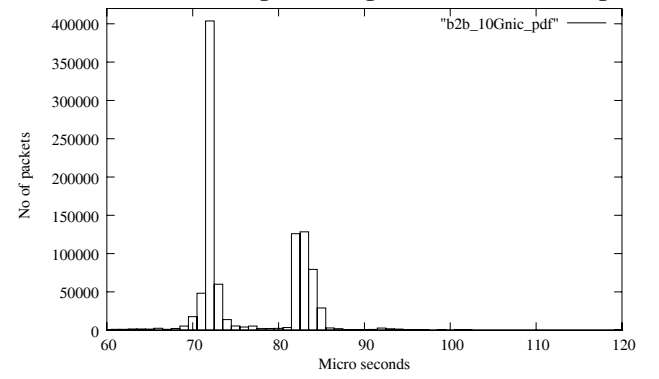

Fig. 5. IPI at senders. Generated on 1 million packets.

work in DRAGON seeks to ease the dynamic provisioning of optical paths; we are also building on UltraGrid to develop congestion control algorithms for interactive video. Design of real time networked applications that can take advantage of provisioned paths, yet operate safely on best effort networks, remains the key challenge in making effective use of hybrid IP/optical networks. Future developments in DRAGON and UltraGrid aim to solve this problem.

\section{ACKNOWLEDGMENTS}

This work is supported by NSF grants 0230738,0334182 . We also thank the DRAGON and HOPI teams for enabling the SC2005 demo.

\section{REFERENCES}

[1] i2CAT Foundation: HD over IP. http://hdwiki.i2cat.net/.

[2] D. D. Clark and D. L. Tennenhouse. Architectural considerations for a new generation of protocols. In Proc. ACM SIGCOMM, Philadelphia, Sep. 1990.

[3] Chelsio Communications. N110 10 gigabit Ethernet adapter.

[4] L. Gharai. RTP Profile for TCP Friendly Rate Control. IETF, October 2005. Work in progress.

[5] L. Gharai, G. Goncher, C. S. Perkins, D. Richardson, and A Mankin. RTP Payload Format for SMPTE 292M. IETF, Mar. 2003. RFC 3497.

[6] L. Gharai and C. S. Perkins. RTP payload format for uncompressed video, Sep. 2005. RFC 4175.

[7] I-Visto. Internet Video Studio System for HDTV Production.

[8] Korean Advanced Institute of Science and Technology. HDTV project. http://cosmos.kaistac.kr/hdtv. (a) IPI for Internet 2 best-effort IP path with 1 Gbps NIC.

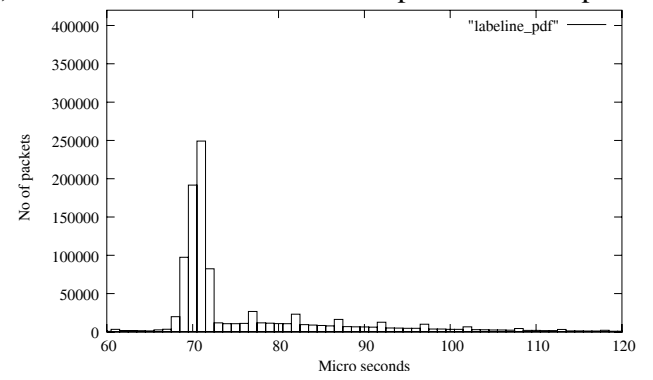

(b) IPI for Internet2 HOPI path with 10 Gbps NIC.

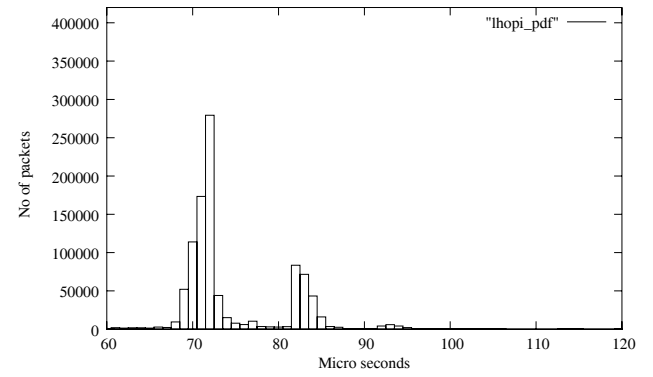

Fig. 6. IPI at receivers. Generated on 1 million packets.

[9] NTT Innovation Laboratories. Uncompressed HDTV transmission system over the internet. NTT Press Release, Oct. 2001. http://www.ntt.co.jp/news/news01e/0110/011026.html.

[10] Argonne National Laboratory. The AccessGrid toolkit, version 2.4. http://www.accessgrid.org.

[11] T. Lehman, J. Sobieski, and B. Jabari. DRAGON: A Framework for Service Provisioning in Heterogenous Grid Netwo rks. IEEE Communications, 44(3), March 2006.

[12] S. McCanne and V. Jacobson. vic : A flexible framework for packet video. In Proc. ACM Multimedia, Boston, Nov. 1995.

[13] Society of Motion Picture and Television Engineers. Bit-serial digital interface for HD television, 1998. SMPTE-292M.

[14] University of Washington. Internet HDTV project website. http://www.washington.edu/hdtv/.

[15] CESNET: The Czech NERN Operator. HD over IP multipoint conference. https://sitola.fi.muni.cz:443/igrid.

[16] C. S. Perkins. RTP: Audio and Video for the Internet. AddisonWesley, June 2003.

[17] C. S. Perkins and L. Gharai. UltraGrid: a high definition collaboratory, Nov. 2002. http://ultragrid.east.isi.edu/.

[18] C. S. Perkins, L. Gharai, T. Lehman, and A. Mankin. Experiments with delivery of HDTV over IP networks. In Proc. the 12th Intl. Packet Video Workshop, Pittsburgh, Apr. 2002.

[19] H. Schulzrinne, S. Casner, R. Frederick, and V. Jacobson. RTP: A transport protocol for real-time applications. IETF, July 2003. RFC 3550.

[20] DVS Digital Video Systems. Centaurus HDTV capture cards. http://www.dvs.de/english/products/.

[21] Tektronix, Inc. The universal network access system (UNAS). http://www.tek.com/Measurement/Products/press/hdtv/. 